\title{
Gobernanza en salud pública: hacia un marco analítico
}

\author{
Governance in Public Health: towards an analytical framework
}

\author{
Marc Hufty \\ Instituto de Altos Estudios Internacionales y de Desarrollo. Ginebra, Suiza. Marc.Hufty@graduateinstitute.ch
}

Recibido 30 Noviembre 2009/Enviado para Modificación 8 Diciembre 2009/Aceptado 28 Diciembre 2009

\section{RESUMEN}

En este texto se argumenta que el "Marco Analítico de la Gobernanza" (MAG) es una metodología con un fuerte potencial para la investigación en políticas sociales, particularmente en el caso de la salud pública. Definiendo el concepto de gobernanza de manera pragmática, ésta se refiere a los procesos de interacción entre los actores involucrados en un asunto público, que llevan a la toma de decisiones o a la formulación de normas sociales. Se postula que hay procesos de gobernanza presentes en cualquier sociedad y, a diferencia de otros acercamientos, éstos se pueden observar y analizar a partir de una perspectiva que no sea normativa o prescriptiva. Finalmente, se desarrolla una metodología apoyada en varias disciplinas sociales que sirve para diagnosticar los procesos colectivos, misma que a su vez, se basa en cinco unidades analíticas principales presentadas aquí: problemas, actores, normas, procesos y puntos nodales. Estas unidades analíticas pretenden constituir una metodología coherente y lógicamente articulada y se propone su uso como instrumento metodológico para la investigación en políticas sociales.

Palabras Clave: Gobernanza, gobierno, salud pública, métodos, política, decisiones, normas (fuente: DeCS, BIREME).

\section{ABSTRACT}

This paper argues that the governance analytical framework (GAF) is a methodology having strong potential for investigation in social policy, particularly in the case of public health. It defines the concept of governance in a pragmatic way as being the processes of interactions between the actors involved in a public affair leading to decision-making or to formulating social norms. It is postulated that any society has governance processes and, unlike other approaches, that these can be observed and be analysed from a non-normative perspective. A methodology for analysing governance processes based on five main analytical units is presented here: problems/stakes, actors, norms, processes and nodal points. These logically articulated analytical units make up a coherent methodology aimed at being used as a tool for social policy research.

Key Words: Governance, government, public health, method, politics, decision theory, decision-making (source: $M e S H, N L M$ ). 
$\mathrm{E}$ 1 campo de la "gobernanza en salud pública" sufre de dos fallas que a mi juicio impiden tener claridad en el razonamiento: una ausencia de coherencia conceptual en el uso de la palabra "gobernanza" y, de manera más general, la "satelización" de las ciencias sociales (CCSS).

Es notoria la ausencia de coherencia conceptual en el uso de la palabra gobernanza (1). Además, tiene una pronunciada afinidad con la normatividad, al ser utilizada para presentar cómo deberían ser las cosas y no cómo son. Amenudo no se concibe como una herramienta heurística de diagnóstico, sino como un impreciso sinónimo de lo político y un pretexto para presentar soluciones exante. Por un lado, esa situación se debe al uso impreciso del concepto, dificultad que se discutirá en la primera parte del texto y por el otro, se le atribuye al hecho de que el campo de la salud pública apunta por esencia a resolver problemas.

Frecuentemente, uno sabe de antemano cual sería la situación ideal y busca lograrla, apoyándose en una investigación en caso de ser necesario. Por ejemplo, frente a brotes de poliomielitis (una situación indeseable), las campañas de vacunación a larga escala aparecen por experiencia como la solución más viable; el trabajo de investigación en gobernanza consiste en identificar los factores políticos que limitan la eficiencia de la campaña (mala organización de los servicios sanitarios, etc.) y una vez identificados, busca resolverlos. En este caso, las CCSS juegan el rol instrumental para entender los obstáculos y la manera de sobrepasarlos.

El problema de esa visión es que se basa en un paradigma mecanicista, una ingeniería social que deja de lado los aportes más enriquecedores de las ciencias sociales. Quizá tiene que ver con el hecho de que en el campo de la salud pública, la mayoría de los problemas que deben resolverse están determinados por las ciencias médicas y el aporte de las CCSS es un anexo. Esto favorece las dimensiones más técnicas de las CCSS, o las dimensiones de las CCSS que presentan afinidad con el paradigma positivista.

Lo anterior hace eco en la tensión aún irresuelta en las CCSS, donde una parte busca acercarse al ideal científico de las ciencias exactas (hay leyes sociales como hay leyes naturales y sólo falta descubrirlas) y otra asume una diferencia intrínseca (la verdad es metodológicamente auto-justificadora y las "leyes" sólo tienen validez en un paradigma definido). De hecho, el razonamiento en CCSS se ubica en varios planos y éstos aportan dimensiones cuya importancia no aparece a primera vista, por ejemplo, las implicaciones 
de las relaciones de poder o el papel de las instituciones en la definición de los problemas y sus soluciones, etc.

A menudo, los factores políticos son percibidos como obstáculos para la solución de problemas, más que como una expresión de la complejidad social. Así entonces, una "buena gobernanza" sería una política "neutral" que facilitaría la solución de tales problemas. Es el sueño del "Estado facilitador", aunque es obviamente una postura irrealista.

En las sociedades complejas existen actores con intereses contradictorios, incluso algunos que se aprovechan de situaciones trágicas y que están preparados para defender a todo precio sus intereses, no siempre económicos, sino ideológicos o irracionales. No sólo se proponen ganar espacio en la cancha, sino que también tratan de influir sobre la definición misma de las reglas del juego.

Entonces, ¿Quién define el problema que hay que solucionar o entender? Es relativamente sencillo determinar el problema y la solución para un brote de poliomielitis; pero, ¿Qué pasa cuando por ejemplo, distintos modelos de salud, aplicados por distintos segmentos del sistema de salud en un mismo país, cruzándose horizontal y verticalmente y compitiendo entre ellos, no se colocan sobre el mismo plano para hacer frente a un problema tal como el aborto? ¿Qué pasa, cuando un primer modelo se basa en la religión para prohibirlo, incluso contra la ley, un segundo promueve respetar estrictamente la palabra de la ley, un tercero busca ante todo el beneficio financiero, y un cuarto se basa en criterios humanistas? Y aún cuando todos los protagonistas de una situación quisieran solucionarla, puede ser que no lo logren y que la suma de acciones individualmente racionales, resulte poco óptima.

La razón conoce sus límites. La gobernanza en la salud pública es una cuestión que debe entonces ser considerada, a partir de un razonamiento que va más allá de las consideraciones técnicas (la aplicación de procedimiento eficientes para una cierta finalidad). Compararía el pensamiento necesario para alcanzar una perspectiva realista en la CCSS con una muñeca rusa (matriochka) donde lo que aparece como un "hecho" depende de las observaciones, de las técnicas de observación, de las cuestiones que se plantean, del contexto social en el cual se seleccionan las preguntas y las respuestas. ¿Es demasiado complicado eso? ¿Le estoy buscando cinco patas al gato? Bienvenidos a las ciencias comprensivas, constructivistas, críticas, no lineales y... realistas. 


\section{¿Gobernanza?}

La finalidad de la primera sección es darle mejor coherencia al concepto de gobernanza (sinónimo de "gobernancia") y para esto, partiré de unas preguntas simples: ¿Qué utilidad concreta (valor agregado) tiene el concepto de gobernanza para el estudio de las políticas sociales?, ¿Cómo hacerlo pasar de un significado borroso a una herramienta analíticamente útil? (2).

La confusión alrededor del término de gobernanza se extiende a tres niveles. Con relación a su coherencia externa, no se diferencia claramente de términos cercanos tal como el de "gobernabilidad", "gobierno" o "gobernación" y esa confusión tiene una raíz histórica (3). Por otro lado, siguiendo al filósofo Peirce (4), una idea clara se reconoce siempre, no se confunde con otra y un término es científicamente pertinente cuando aporta un cambio a nuestra comprensión de un fenómeno. Se necesita entonces una definición exacta y discreta del concepto, así como la identificación de sus parónimos. Con relación a su coherencia interna, no existe una definición consensual y estable de la gobernanza, es polisémica. No sólo se utiliza el término para diferentes objetos y con definiciones distintas, pero algunos lo utilizan con varios sentidos en diferentes obras, y a veces en un mismo texto. La solución se puede dar con una definición precisa y con un objeto claramente delimitado. Con referencia a su coherencia social, se trata de un concepto políticamente cargado o polémico, porque se utiliza como herramienta para imponer desde afuera un cambio social (en América Latina, se usa el termino de "buena gobernabilidad"). En este caso, la solución se logra con una distinción explícita entre propuestas analíticas y normativas.

Para resolver provisionalmente la cuestión de la coherencia externa, sobre todo en el contexto que nos ocupa, gobernar es manejar las políticas o los asuntos de una organización. La gobernación puede ser la acción de gobernar, un Ministerio (del interior en México entre otros) o una entidad territorial subnacional (manejada por un Gobernador). El Gobierno es el conjunto de los ministros de un Estado, mientras que la gobernabilidad se refiere a la calidad de ser gobernable (5), a la capacidad de un sistema sociopolítico de controlarse (6), o más precisamente a "un estado de equilibrio dinámico entre el nivel de las demandas sociales y la capacidad del sistema político para responder a ellas de manera legítima y eficaz" (7).

A continuación, haré referencia de manera sintética a los usos más comunes del concepto, identificando sus aportes y limitaciones, para luego desarrollar mi propuesta. 
Con base en lo que se observa en la literatura y en la práctica, se usa el concepto de acuerdo a dos grandes perspectivas: como un marco normativo apuntando al cambio social o como un marco de análisis que explore los sistemas de coordinación no jerárquicos. En este caso cuenta con las siguientes características: los actores y lugares para la toma de decisión son múltiples, son formales (reconocidos por una autoridad) tanto como informales y las relaciones entre los actores son horizontales más que verticales, con una tendencia a ser autorreguladas.

Cada corriente presentada aquí combina la perspectiva analítica (examina detalladamente un fenómeno para explicarlo, apoyándose en ciertos elementos teóricos y metodológicos) con la perspectiva normativa (pretende resolver una clase de problemas). Con la finalidad de desarrollar una definición precisa con un objeto claramente delimitado, propongo evaluar los enfoques presentados mediante los siguientes criterios: 1. Valor agregado (¿Este enfoque aporta algo más de lo que ya existe?), 2. Cientificidad (¿Es observable empíricamente, refutable y generalizable?), 3. Operatividad (¿Se apoya en una metodología reproducible?).

\section{La gobernanza de organizaciones}

La primera corriente apareció en los Estados Unidos con el desarrollo de las ciencias de la administración y de la organización. Se observa desde principios del siglo XX, con los trabajos de Elton Mayo, que la jerarquía formal en una empresa no corresponde necesariamente a la jerarquía efectiva y que las relaciones entre empleados son determinantes en la organización del trabajo. Se sabe también, con base en los trabajos de Ronald Coase, que la reducción de los costos de transacción (por ejemplo, el costo de reunir la información necesaria para tomar una decisión) motiva la creación de organizaciones jerárquicas, pero también que por encima de un cierto nivel de complejidad (multiplicación de los actores y unidades, creciente especialización, etc.), los costos administrativos internos aumentan y pueden sobrepasar el beneficio de esa reducción. Desde la perspectiva de la gobernanza (corporate governance), una organización constituye un espacio de cooperación y conflictos entre varias clases de actores (empleados, ejecutivos, dueños, reguladores, etc.), con sus normas, intereses y prácticas, y se caracteriza por tres clases de mecanismos de coordinación: los verticales, horizontales e informales.

A partir de los años 1970, se estudiaron particularmente dos tipos de organizaciones: las universidades (8) y las empresas, con la preocupación 
por una parte de mantener la eficiencia de las organizaciones complejas, pero también de resolver la discrepancia creciente entre la propiedad y el manejo.

Se trataba por lo tanto de observar y teorizar los mecanismos e instituciones que permitían la coordinación entre las unidades de una organización, el arbitraje de las divergencias, el mantenimiento de la cohesión del conjunto, la minimización de los costos de transacción y el diseño de organizaciones más eficientes. Los trabajos de la escuela institucionalista, en particular de Oliver Williamson (9-11), Premio Nobel 2009, demostraron el rol central de la gobernanza a partir de los conceptos de transacción, contratos, reglas de juego e instituciones, implicando una crítica mayor a la teoría económica neoclásica que descuidó el rol de las organizaciones e instituciones.

Buscando mejorar la gobernanza de las empresas, apareció una reflexión normativa sobre la buena gobernanza de empresas. Por ejemplo, los "Principios de Gobierno de Empresa de la OCDE", una serie de criterios ideales apuntando a "elaborar una base común, indispensable para la aparición de prácticas de calidad en el ámbito de la gobernanza de la empresa" (12). Son propuestas fuertemente influenciadas por la práctica anglo-sajona.

En resumen, los trabajos alrededor de la gobernanza de organizaciones hicieron algunos aportes teóricos y metodológicos determinantes. Por el contrario, las tentativas para establecer criterios de buena gobernanza de empresas se ubican fuera del campo científico.

\section{La gobernanza global}

Los razonamientos institucionalistas encontraron eco en las relaciones internacionales, una subdisciplina de la ciencia política. Según el enfoque tradicional, no existe formalmente en el sistema internacional una entidad supraestatal que pueda por ejemplo, arbitrar los conflictos. La coordinación entre los Estados sería entonces horizontal, dando un espacio natural a una visión de gobernanza y permitiendo de esta manera, comprender el proceso de elaboración de acuerdo con el objetivo de solucionar problemas de coordinación fuera de la autoridad centralizadora de un Estado (13).

La visión de una sociedad internacional sin jerarquía era obviamente una ficción si se consideraba el poder hegemónico de algunos de ellos, pero varios cambios modificaron el análisis. La vida internacional se institucionalizó: se 
crearon una multitud de acuerdos internacionales y de "regímenes internacionales" (14). El número y las categorías de los protagonistas presentes sobre la escena internacional se multiplicó y nuevos actores crearon espacios de acción "multi-niveles" o "policentricos", actuando tanto al nivel local o nacional que global, y tomando responsabilidades que pertenecían anteriormente a los Estados. El resultado de este fenómeno resultó en una situación de gobernanza global, definida de la siguiente manera por la Comisión sobre la Gobernanza Global (15):

"El conjunto de los distintos medios por los cuales los individuos y las instituciones, públicas y privadas, administran sus asuntos comunes. Es un proceso continuo de cooperación y compromisos entre intereses diversos y conflictivos. Incluye las instituciones oficiales y los regímenes dotados de poderes ejecutorios, así como los acuerdos informales sobre los cuales el pueblo y las instituciones cayeron de acuerdo o que perciben ser de su interés."

En las relaciones internacionales, esa visión constituye un avance impresionante, pues se considera el conjunto de los protagonistas ya sean oficiales o no, se integran los acuerdos tanto formales como informales y es procesual o dinámica.

Este enfoque analítico se acompañó de su versión normativa (y no científica). Debido a que el desorden es desfavorable al progreso económico del cual depende la prosperidad común, la Comisión buscaba, después de la caída del Muro de Berlín, promover un nuevo orden mundial, haciendo hincapié en el consenso y descuidando la realidad de las relaciones de poder en las relaciones internacionales.

\section{La Gobernanza Moderna}

A fines de los años 1970 se ha podido observar un creciente cuestionamiento del rol del Estado en los países industrializados (16). En síntesis, se percibía una crisis de gobernabilidad. El éxito económico de la posguerra había dado lugar a una intervención creciente del estado en la sociedad, pero también a una aumentación incontrolable de las demandas y a una reducción creciente de su margen de maniobra.

El crecimiento del aparato estatal había creado múltiples redundancias e ineficiencias a la imagen de las organizaciones complejas. La globalización (aceleración de los transportes y de la comunicación, circulación acelerada de los capitales, uniformización de los modos de consumo, procesos de integración regionales, internacionalización de las empresas, etc.) redujo el espacio de influencia de las políticas públicas. 
Para Jessop (17), existen tres clases de mecanismos de regulación de la sociedad: jerárquico (por la autoridad), económico (por el mercado) y heterárquico (por redes autoorganizadas y asociaciones). Estos mecanismos coexisten siempre, pero en configuraciones variables. En esos años se redujo la importancia de la regulación jerárquica a favor de la económica y heterárquica. Muchos mecanismos de producción o regulación de los bienes públicos se desarrollaron fuera del Estado, a niveles subestatales o supraestatales. Nuevas organizaciones, nuevos actores y "redes autónomas" reemplazaron al Estado en varias de sus funciones anteriores.

Para analizar ese fenómeno, algunos autores como Kooiman (18) y Rhodes (19) desarrollaron el concepto de gobernanza moderna (modern governance), dando lugar a una reflexión centrada en la interrogación de la gobernanza de organizaciones y de las relaciones internacionales: ¿Cómo lograr la coordinación eficiente de actores múltiples sin que sea por medio de una autoridad vertical?

Sin embargo, las hipótesis del vaciamiento (hollowing out) o debilitamiento del Estado todavía quedan por demostrarse. Varios analistas señalan que al concentrarse en sus funciones principales, el Estado por el contrario se reforzó, incluso con relación a los protagonistas con fama de autonomía (20, 21). Además, ese análisis se basa esencialmente en estudios realizados en los países industrializados y sobre un período de tiempo relativamente corto.

El concepto de gobernanza moderna puede exportarse difícilmente a los países en desarrollo y si bien se hicieron estudios rigurosos en ciertos aspectos de los cambios mencionados, mucha literatura queda a un nivel muy general. No se ha logrado aún desarrollar una definición o una metodología precisa de la gobernanza moderna. Una de las razones fue quizás, su relación ambigua con la fase ideológica que caracterizó a los años ochenta.

Precisamente, resulta difícil diagnosticar entre ese fenómeno y las políticas de los gobiernos conservadores (Thatcher, Kohl y Reagan) lo que fue la causa y la consecuencia. Por ejemplo, el Gobierno británico estimuló sistemáticamente la privatización de los servicios estatales, la reducción de su espacio de competencia y la aplicación de los principios de la gestión de empresas privadas en el sector público (nueva gestión pública) (22). Hizo de la gobernanza moderna un programa político que se presentaba como una solución a la crisis de gobernabilidad del Estado: la gobernanza por las redes iba a sustituir a las soluciones jerárquicas y el Estado se satisfacería con un 
papel de "facilitador" en una democracia de mercado. Sin minimizar la pertinencia de las interrogaciones de esa corriente o sus impactos, hoy en día con el regreso del Estado se evidencian sus limitaciones.

\section{La buena gobernabilidad}

En su cruzada por mejorar la situación económica de los países en desarrollo, el Banco Mundial (BM) tuvo que profundizar sus técnicas de intervención en los límites de su mandato, el cual le prohíbe una intervención política directa. Llegó a la conclusión de que el éxito o fracaso de las políticas de ajuste estructural dependía de actores políticos no gubernamentales. El corolario de esa observación fue buscar una herramienta metodológica destinada a identificar los lugares de poder efectivos en los sistemas políticos, que sean formales o informales, estatales o no, y entonces encontró, a fines de los ochenta, el concepto de gobernanza, que definió de la siguiente manera: "La gobernanza es la manera a través de la cual se ejerce el poder en la gestión de los recursos económicos y sociales para el desarrollo de un país" (23).

Sin embargo, el problema real era que en su visión, numerosos gobiernos no actuaban de manera óptima a favor del desarrollo. Así entonces, el BM desarrolló una serie de criterios que permitían el análisis y la evaluación de las prácticas de los Estados en cuanto a la gobernanza a través de la precisión de metas con las cuales se evaluaba el grado de cumplimiento de los Estados. La gobernanza pasó de ser una herramienta analítica (governance) a ser una herramienta política de transformación de las sociedades (good governance). Su uso tomó una connotación normativa, que se refiere a lo que "debería ser" y no a lo que "es". Sin importar cuales sean esos criterios (de hecho provienen de los códigos morales y modelos políticos de los países industrializados), cuando se imponen desde afuera, no pueden sino ser percibidos como parte de una relación de poder. Por otra parte, desde el punto de vista epistemológico, los criterios propuestos no son refutables in fine. Nada permite juzgar entre dos opciones normativas, sino valores. La buena gobernabilidad se ubica claramente en el campo político.

Asimismo, agregó dos consideraciones adicionales. Dadas esas primicias, se puede desarrollar una metodología científica para observar las discrepancias entre los criterios de buena gobernabilidad y las prácticas $(\mathrm{Ej}$. la transparencia de los procedimientos de adjudicación de un mercado público). De hecho, se dedican recursos importantes al desarrollo de tales metodologías (24). Luego, la aplicación de la gobernanza como elemento de condicionalidad para las agencias de cooperación causó mucha confusión conceptual y 
resistencia, especialmente en América Latina. El hecho afortunado fue que good governance haya sido traducido por "buena gobernabilidad" y no por "buena gobernanza" y quizá entonces, se puede todavía salvar el concepto como herramienta analítica.

\section{El marco analítico de la gobernanza}

En respuesta a la necesidad de una metodología para el análisis de la gobernanza en la salud pública y con base en las corrientes descritas, desarrollé una propuesta original, el Marco Analítico de la Gobernanza (MAG).

La gobernanza se refiere aquí a una clase de hechos sociales, los procesos colectivos formales e informales de toma de decisión y de elaboración de normas sociales con relación a asuntos públicos.

Bajo esta visión, es un concepto generalizable. En toda sociedad, sea local, nacional o internacional y en cualquier momento de su historia, se pueden observar procesos de gobernanza. No es un fenómeno limitado a nuestra época. En cualquier sociedad, aún en las sociedades sin Estado (por ejemplo indígenas, o zonas de guerra civil), se deben tomar decisiones y se necesitan sistemas de reglas para la toma de decisión. Existen normas sociales (legales o consuetudinarias) e instituciones (sistemas recurrentes de normas sociales) que permiten de poder vivir juntos. No es equivalente al sistema político, lo cual caracteriza una forma específica de sociedad, el Estado-Nación, Tampoco es equivalente a las políticas públicas, que son actas del Estado central.

No es un concepto normativo o prescriptivo. La gobernanza no es por lo tanto, un modelo que sea deseable fomentar. Cada sociedad desarrolla sus modos de gobernanza, sus sistemas de toma de decisión o de resolución de conflictos entre sus miembros, sus normas e instituciones. Aquí se trata de desarrollar una metodología para analizarles sin juzgar. Obviamente en epistemología, toda teoría se apoya in fine sobre valores (Ej. uno analiza el poder porque piensa que un mejor conocimiento de los mecanismos sociales puede conducir a una sociedad mejor), pero mi ambición aquí es desarrollar una propuesta utilizable por personas con visiones distintas y hasta sin conocimiento previo de las ciencias sociales. Cuando uno está frente a una situación social que define como gobernanza, necesita, para poder comprenderla, una metodología que sirva de referencia a su observación, descripción y análisis. 
Mi ambición es proponer tal metodología. Los procesos de gobernanza de distintas sociedades, en distintos lugares y tiempos, toman distintas características que pueden analizarse con ayuda de esa metodología, entendiendo un marco analítico como un conjunto coherente de modelos (representaciones esquemáticas) asociado a una metodología que permita el pasaje entre generalizaciones teóricas y observaciones empíricas.

Con base en la discusión anterior sobre las corrientes de gobernanza, esa metodología tiene que presentar las siguientes características: realista, interdisciplinaria, generalizable, comparativa, reflexiva y operacional. Realista indica su capacidad para describir los hechos tal como son, en oposición a las perspectivas normativas. La interdisciplinariedad se refiere a una propuesta que utiliza conceptos y técnicas procedentes de disciplinas diferentes para mirar a un objeto común. La gobernanza sería un concepto pasarela (25) entre disciplinas. Generalizable y comparativa significa que se puede observar cualquier modo de gobernanza y comparar con otros basándose en las categorías analíticas que propone. La reflexividad se refiere al efecto del observador. En un primer nivel, observar hechos les modifica, especialmente en ciencias sociales. En un segundo nivel, el observador realiza una selección de los hechos que observa, así como de la metodología y de las interpretaciones. Esto implica la consideración del proceso de gobernanza observado tanto por el observador como por el actor, así como la medición de las implicaciones que este proceso representa. En la perspectiva de su operacionalidad, una metodología de observación de los procesos de gobernanza debe permitir el análisis de hechos concretos, así como la ayuda para resolver problemas reales.

El marco analítico propuesto consta de cinco categorías analíticas: los problemas, los actores, los puntos nodales, las normas y los procesos. Los problemas son los que están en juego. Los actores son individuos o grupos cuya acción colectiva conduce a la formulación de normas (reglas de juego) que orientan el comportamiento de los actores. Las interacciones se pueden observar en puntos nodales, espacios físicos o virtuales de interfase en donde convergen los problemas, procesos, actores y normas. Los procesos son sucesiones de estados por los cuales pasan las interrelaciones entre actores, normas y puntos nodales. Los procesos, los actores y las normas pueden ser formales, es decir, reconocidos por los actores portadores de autoridad en la sociedad observada (este reconocimiento es legal al nivel de las sociedades dotadas de un derecho positivo), o informales, definidos por la práctica de los actores. 


\section{Definición del problema}

En general, el uso del MAG supone dirigirse a problemas que tienen que ver con la gobernanza tal como está definida aquí, es decir para los cuales se pueden aislar algunas características analizables con esa metodología, por ejemplo, inequidad en el acceso a la salud pública.

La primera etapa de aplicación del MAG sería entonces la de definir con claridad el problema y lo que está en juego. No es nada inocente. Desde una perspectiva constructivista, todo problema es una construcción social y si se acepta que un problema es siempre una discrepancia entre una situación existente y una deseada, el problema es entonces por definición, enteramente subjetivo. Puede ser que haya consenso entre muchas personas sobre un problema, pero no deja de ser una cuestión de percepción, pues ésta depende de los valores de quienes identifican una situación como un problema. De hecho, el problema de alguien puede ser una suerte para otros. Si se toma por ejemplo el caso de la gripe A H1N1, vemos que es evidentemente un problema de salud pública, pero para las empresas farmacéuticas productoras de vacunas es una bonanza.

Otro ejemplo sería el de un bosque bajo protección en el centro de demandas incompatibles y conflictivas. Puede ser que la deforestación sea el problema mayor para los ecologistas, pero para los campesinos sería tener tierra y dar de comer a sus hijos, para los indígenas, el mantenimiento de la integridad territorial y de la cultura, para los madereros, el acceso a la madera para ganarse la vida, para una empresa petrolera, el acceso a los recursos minerales del subsuelo y para el estado, el equilibrio entre el desarrollo económico, conservación y paz social, etc.

Entonces, lo que está en juego puede ser absolutamente diferente para cada protagonista debido a que cada quien tiene su propia percepción del problema (incluso el observador quien está utilizando el MAG para hacer un diagnóstico). Esto se explica por el lugar ocupado en la sociedad, las historias, culturas, creencias, los apremios y objetivos, en resumen los "universos de significación". Cualquier interacción social es un encuentro entre universos de significación distintos. Al emprender un estudio con el MAG, tenemos ante todo que admitir esa pluralidad de visiones, lo que implica distanciarnos de nuestra perspectiva como observador o actor. Es un paso fundamental para un análisis realista y una solución posible. Por lo tanto, la primera etapa del MAG es la de deconstruir y reconstruir el problema. 
La identificación del problema hace parte en sí misma de lo que está en juego y es a menudo objeto de un proceso de gobernanza cuando varios protagonistas quieren imponer sus agendas. A menudo, pero no siempre, el actor con más recursos logra imponer su agenda, ya sea por la fuerza, la persuasión o la violencia simbólica (convenciendo a otro que sus preferencias son iguales a las del actor dominante, como en la relación patrón-cliente) (26). Así entonces y con probabilidad elevada, la imposición dejará descontento y conducirá a formas pasivas o activas de resistencia siendo ésta misma, una causa mayor de fracaso de los proyectos de cambio social.

Al nivel práctico, existe una manera simple de hacer las cosas para reconstruir un problema. Se toma una hoja de papel, se escribe todo lo que a uno le viene a la mente cuando piensa en tal problema y luego se elaboran una serie de categorías para ubicar las distintas ideas. Este ejercicio nos permite desarrollar nuestra propia concepción de un objeto, incluso si lo hacemos con honestidad, podremos concebir nuestros prejuicios. La segunda etapa sería la de hablar y escuchar a los diferentes protagonistas, pues con la ayuda de esas discusiones, de una revisión de la literatura y de nuestro ejercicio reflexivo, podremos reconstruir la problemática.

Pasa a menudo por la transformación de problemas sociales ("mis niños son enfermos y no puedo comprar remedios") en problemas sociológicos (el "acceso a la salud es desigual") y por la puesta en evidencias de los mecanismos sociales subyacentes a la situación estudiada. Para el observador, la capacidad de superar lo que los actores presentan como un problema y convertirlo en una cuestión sociológica depende mucho del contexto, de su metodología, técnicas y experiencia. No debería haber ningún tabú cuando se asume esa etapa.

Obviamente, cuando uno está en un contexto cerrado (el problema esta definido de antemano, como en un mandato) esa etapa es limitada. Pero en este caso, valdría la pena interrogarse sobre la posibilidad de que esa misma limitación sea parte del problema.

\section{Las normas}

Las normas sociales, en una primera aproximación, son las reglas del juego. Están en juego en todos los procesos de gobernanza y esto sucede en tres niveles. En un primer nivel, puede ser que en un debate la reglas sean conocidas y aceptadas por todos y en este caso se reproducen implícitamente las normas; en un segundo 
nivel, puede ser que sean debatidas abiertamente, reproducidas o cambiadas; y en un tercer nivel, lo que está en juego puede ser lo que califico de metagobernanza, es decir, las normas que estipulan cómo se van a definir las reglas y quién tiene la legitimidad de decidir las reglas. En fin, siempre que hay acuerdos y decisiones entre actores dentro de una sociedad, hay por ende formulación, confirmación, invalidación o adaptación de normas como consecuencia de los procesos de gobernanza.

Con más precisión, se pueden definir las normas como "las expectativas colectivas haciendo referencia a lo que es considerado un comportamiento apropiado en una sociedad". Éstas incluyen tres elementos: se apoyan en valores o creencias (el sentido de lo que es bueno o incorrecto), tienen un elemento prescriptivo (sugieren lo que uno tiene que hacer o no), y suponen sanciones positivas o negativas. Por ejemplo, saludar a la gente es una norma social: facilita las relaciones y reproduce la sociedad. Se enseña a los chicos (es el Habitus o el sentido práctico de Bourdieu) que el que no saluda a los conocidos adecuadamente, sufrirá la sanción del ostracismo o de los chismes.

Es preciso entenderlo aquí, desde el punto de vista sociológico. Utilizo la categoría general de "normas sociales" para distanciarme del uso muy común en América Latina que es el de confundir normas con leyes. Las normas sociales sí pueden ser legales (es decir inscriptas en el derecho positivo del Estado, por ejemplo la limitación de velocidad a $100 \mathrm{Km}$. por hora en una autopista), o a un nivel más general, formales (reconocidas por las autoridades de la sociedad estudiada, estatales o no) y hasta informales (creadas por la práctica de los actores sin haber sido codificadas, el hecho de que los conductores manejen casi todos encima del limite legal, a $110 \mathrm{Km}$. por hora, en la misma autopista).

Por lo tanto, las normas influencian (limitan, facilitan) el comportamiento de los actores, pero es importante entender que éstas están constantemente reproducidas, redefinidas, modificadas por la acción colectiva, una idea que se califica de agente-estructura y que soluciona la tensión entre el individualismo y el holismo sociológico.

Cuando los actores internalizan las normas haciendo referencia a un tema (las aceptan o adaptan su comportamiento), se habla de un proceso de institucionalización. El conjunto de las normas que duran en el tiempo (son recurrentes) con relación a un tema determinado, se describen como instituciones sociales. La institución del matrimonio está definida como el conjunto de las normas a su propósito, legales o informales. 
En cada sociedad existen sistemas normativos (instituciones) distintos, promovidos por actores diferentes que coexisten al mismo tiempo, se sobreponen y están en competencia. Esa situación de pluralismo normativo constituye una fuente mayor de conflictividad cuando cada actor o coalición de actores intenta imponer su visión en una relación de poder que pasa por el sistema político formal o informal.

Las normas pueden ser de tres tipos, las cuales a su vez se remiten a diferentes niveles de análisis:

- En primer lugar, las meta-normas se refieren a premisas o principios que orientan el contrato social en su sentido amplio, definiendo criterios y valores estructurales (por ejemplo el principio de participación en la Declaración de Alma Ata o el principio de recuperación de los costos en la Iniciativa de Bamako).

- En segundo lugar, las normas constitutivas $(27,28)$ son las que dan a un actor (individual o colectivo) su identidad y las que para una organización definen los mecanismos organizativos e institucionales relacionados con el funcionamiento sectorial del objeto o escenario bajo análisis (por ejemplo los Estatutos de la OPS o la ley que regula la salud pública en un país).

- En tercer lugar, las normas regulativas, o reglas, son las que definen las reglas de conducta que precisan lo que es apropiado desde el punto de vista de la sociedad en términos de comportamiento, aquello que cada uno debe o puede hacer, y precisan sanciones positivas (aprobación, recompensa) o negativas (desaprobación, castigo), (por ejemplo los reglamentos de una biblioteca o de un hospital).

Las normas expresan también los aspectos multi-niveles de la gobernanza. Pueden ser formuladas en varios niveles y transferidas a otros. Se elaboran a menudo al nivel nacional, eventualmente en una "comunidad epistémica" (29) y se transfieren al nivel internacional y nuevamente a otros países por mecanismos internacionales, donde serán transferidas al nivel local.

En cada nivel, hay un proceso de reacción, de rechazo o de internalización y adaptación. Por ejemplo, la meta-norma de participación local se convirtió en una norma dominante en el conjunto del régimen internacional de la cooperación para el desarrollo. Se trasladó al mundo de la salud a través de los mecanismos de financiamiento internacionales. 
Para analizar los procesos de cambio de las normas sociales se sugiere utilizar una matriz que permita dividir las etapas del proceso de producción, difusión y reacción de las normas, analizar los distintos niveles y reconocer las relaciones mutuas de condicionamiento. Este proceso es ilustrado por el gráfico siguiente. Cabe señalar que los niveles son artificiales. Como todo modelo, este tampoco pretende dar una representación exacta de la realidad, pero sí una aproximación simplificada en función de la necesidad. Por lo tanto, se pueden añadir niveles, por ejemplo el nivel regional subestatal o supraestatal (la región del Noroeste argentino o la región Suramérica).

Figura 1. Matriz de análisis de las normas

\begin{tabular}{|l|c|c|c|c|}
\hline \multicolumn{2}{|c|}{} & \multicolumn{3}{c|}{ Etapas } \\
\cline { 3 - 5 } & Elaboración & Transmisión & Reacción \\
\hline \multirow{4}{*}{ Niveles } & Internacional & & & \\
\cline { 2 - 5 } & Nacional & & & \\
\cline { 2 - 5 } & Local & & & \\
\cline { 2 - 5 } & & & & \\
\hline
\end{tabular}

Los actores

El MAG es una metodología centrada en los actores. Las normas sociales están intimamente ligadas a los actores, sus conductas e interacciones, y condicionadas por sus características: identidad, poder, intereses, ideas e historias. Una diferencia importante que mantiene con algunas metodologías es que toma en cuenta a todos los actores, o stakeholders, ya sean formales o informales (reconocidos o no por la ley o las autoridades), sin prejuicios e incluyendo tanto a individuos como a grupos. Las redes sociales por ejemplo, forman parte de la realidad de cada sociedad organizada, pero no se integran usualmente a un análisis de actores, a pesar de su importancia en muchas decisiones.

Existen diferentes herramientas para analizar a los actores (multistakeholders analysis). Se elabora generalmente un sistema de descripción de cada uno de ellos, adaptándolo a las necesidades de la investigación con la finalidad es establecer una comparación.

Por supuesto, no todos los agentes tienen la misma influencia en un proceso de gobernanza y es por esta razón que propongo herramientas dirigidas a distinguir diferentes clases de actores. Un primer aspecto tiene que ver con 
la importancia de un actor en el proceso de gobernanza estudiado y para esto, también existen diferentes maneras de hacer una clasificación.

Así entonces propongo, con base en los trabajos de Bourdieu (30), un acercamiento a los recursos controlados o movilizados por los actores. En síntesis, el capital simbólico es el prestigio del cual goza un actor en un campo (el prestigio de los médicos en el campo de la salud pública es elevado, lo de los politólogos es no existente), el capital económico es el dinero o las propiedades, el capital social sería la calidad de la red social y el capital cultural es el conjunto de los conocimientos movilizables, ya sean teóricos o prácticos.

Según la teoría de los campos sociales, los actores que tienen control sobre capitales importantes en un campo, son más dominantes que aquellos que controlan menos recursos. Es evidente, por lo tanto, que en el campo de la legislación, los diputados tienen más poder que un campesino.

Existe una relación entre los actores, los recursos y el poder o la capacidad de influenciar sobre la naturaleza de la trama de relaciones, reglas y procedimientos establecidos. El tipo de recursos controlados, la combinación de los mismos por un actor y su habilidad para movilizarlos en función de sus propios intereses potencian su poder para determinar o cambiar las reglas de juego. La metodología propone entonces un análisis situacional del poder relativo de los actores. Es una función de cuatro variables: los recursos controlados, la voluntad y capacidad de movilizar sus recursos, la movilización efectiva en el proceso de gobernanza analizado y la interacción estratégica con los demás actores. Dos dimensiones se tendrían que considerar en una evaluación empírica: la dimensión objetiva de esas variables (por ejemplo de que clase de recursos financieros dispone un actor) y la dimensión subjetiva, la cual depende de la percepción de los actores. Esta última dimensión es sumamente importante, pues la capacidad de aparecer con más poder de lo que uno tiene en realidad, es un recurso determinante en una interacción estratégica.

Sin prejuzgar la metodología utilizada por el observador, proponemos una clasificación de los actores en tres categorías según esas cuatro variables: "actores estratégicos", "relevantes", y "secundarios". El esfuerzo de análisis se concentrará con prioridad en los actores estratégicos. El desarrollo conceptual de Prats (31) permite definirles como "todo individuo, organización o grupo con recursos de poder suficientes para impedir o perturbar el 
funcionamiento de las reglas o procedimientos de toma de decisiones y de soluciones de conflictos colectivos". En cambio, los actores relevantes son actores involucrados en la trama institucional que poseen los recursos necesarios para considerarse como estratégicos, pero que se movilizan o que se ven dominados en el proceso. Los actores secundarios no tienen el poder suficiente para determinar el cambio de las reglas de juego.

Los puntos nodales

Figura 2. Punto nodal

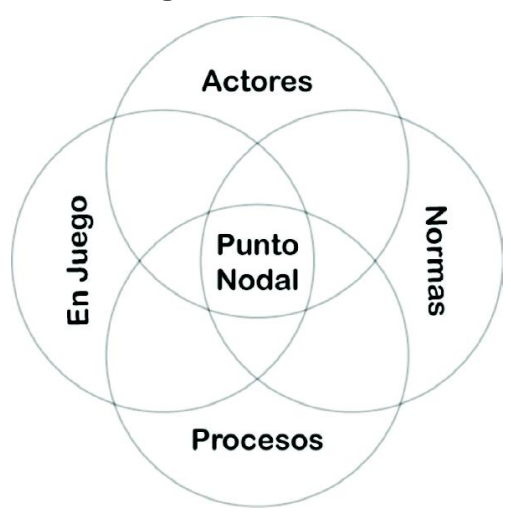

Se entienden por puntos nodales los espacios físicos o virtuales en donde convergen, en un proceso de gobernanza dado, problemas, actores, procesos (tiempo y espacio), en donde se toman decisiones, se concluyen acuerdos y se elaboran normas sociales. Un ejemplo concreto sería una mesa de negociación entre varias entidades, un consejo de administración, el Gobierno, el estado mayor, el consejo de los ancianos, la cúpula de la mafia, etc. Ahí se cruzan universos de significación o visiones del mundo, culturas, discursos o relaciones de fuerza. Identificar puntos nodales es una puerta de entrada ideal para la observación de los procesos de gobernanza.

Un punto nodal se puede componer de varios niveles de puntos nodales. Por ejemplo, en una mesa de negociación con el Gobierno (B), el patronato (C) y un mediador (D), está representado el movimiento sindical (A). El "movimiento" se compone de varios sindicatos (A1, A2 ...), y cada sindicato de varios entes (A11, A12...). Es una cadena de puntos nodales, tal y como se ilustra aquí. 
Figura 3. Cadena nodal

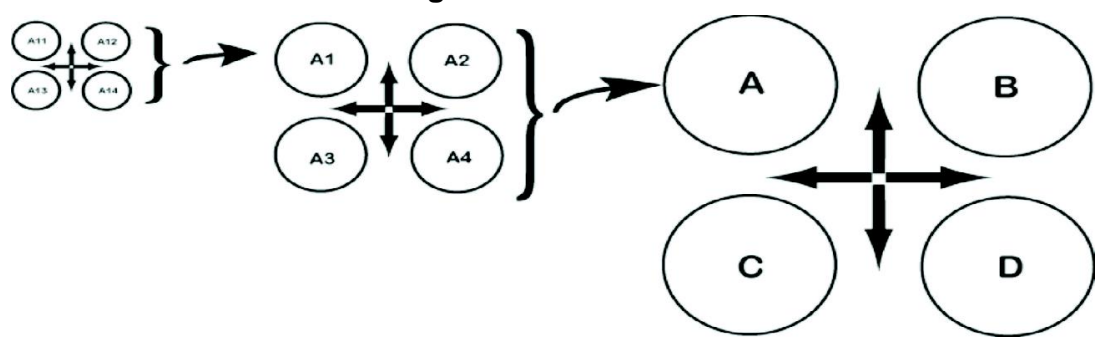

El nivel que se quiere observar o los puntos nodales que se debe estudiar dependen del problema observado. Claro que para entender cómo el movimiento sindical logró conformar la propuesta que está presentando en la mesa, el observador tendrá que estudiar varios niveles de puntos nodales en el movimiento sindical.

Este análisis pretende reconocer los puntos nodales formales e informales que forman parte de la trama de espacios decisorios. La identificación de diferentes puntos nodales, su caracterización, las relaciones y sus efectos sobre las variables dependientes permiten analizar las condiciones existentes (favorables o desfavorables) para un proceso de cambio.

\section{Los procesos}

Los procesos son sucesiones de estados por que pasa un sistema. Las situaciones evolucionan, los procesos retroceden a veces, pero son dinámicos. La idea de proceso introduce la historicidad de los modelos de gobernanza. De esta forma, es posible para un objeto dado, o un puntonodal, identificar secuencias que permitan evaluar la dirección por la cual evolucionan esos procesos y de localizar los factores favorables al cambio. El análisis de los procesos de cambio busca identificar los patrones de evolución de los puntos nodales, la trama de interacciones entre actores y su relación con los cambios de las reglas de juego a través del tiempo (representado aquí por una flecha).

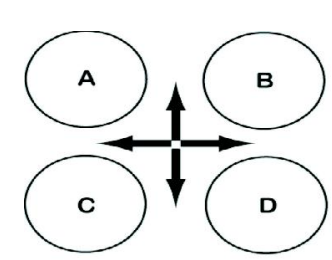

Figura 4. Proceso

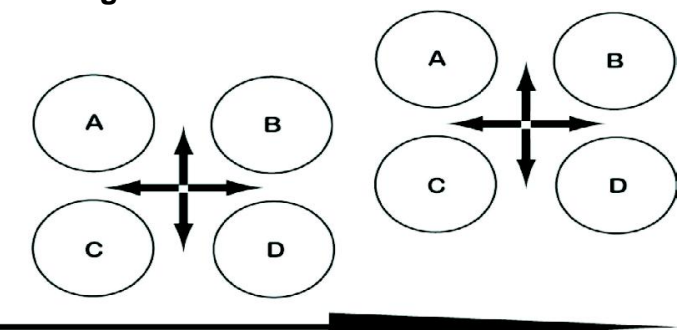




\section{Operacionalidad}

En la perspectiva de su operacionalidad, el MAG debe permitir el análisis de hechos concretos y ayudar a resolver problemas reales, pero también deberia poder ayudar a resolver problemas concretos una vez que se identifican las relaciones entre los varios elementos de un proceso que se analizan. Esto depende de las preguntas e hipótesis del que la utiliza.

Se supone que el problema seleccionado se puede estudiar desde el MAG, es decir que se pueden aislar en el problema algunas características analizables con esa metodología. Puede haber múltiples factores causales (multi-causalidad). Si se acepta la perspectiva explicativa, un proceso de gobernanza está influenciado por varios factores relacionados al contexto social, político e histórico, etc. y entonces se considera ese proceso como un elemento de una cadena explicativa. Es una variable intermedia y es a la vez causal y causada.

Figura 5. El proceso de gobernanza como una variable intermedia

\begin{tabular}{|c|c|c|c|}
$\begin{array}{c}\text { Variables independientes } \\
\text { (contexto) }\end{array}$ & $\begin{array}{c}\text { Variables dependientes } \\
\text { (problema) } \\
\text { Categorias analiticas }\end{array}$ \\
\hline
\end{tabular}

Así por ejemplo, si el problema identificado es la inequidad en el acceso a los servicios de salud pública, medida para algunas categorías sociales (a pesar de la ley que garantiza la equidad), esa inequidad está siendo reforzada día con día por los procesos de gobernanza, pero éstos dependen de la organización institucional del sistema, de la historia, etc.

El MAG permite descomponer esos procesos según los puntos nodales y observar cada punto nodal para entender exactamente como las reglas de acceso a la salud pública se interpretan en la práctica y conducen a normas informales de inequidad más potentes que la ley. En la Figura 6 se presenta un ejemplo simplificado que explica cómo entre cinco puntos nodales que componen el proceso de gobernanza, se identificó el punto nodal E como causal en el problema: será el foco de intervención (estudio y cambios de normas) para lograr un mejoramiento del problema bajo estudio (la inequidad). 
Figura 6. Metodología de intervención

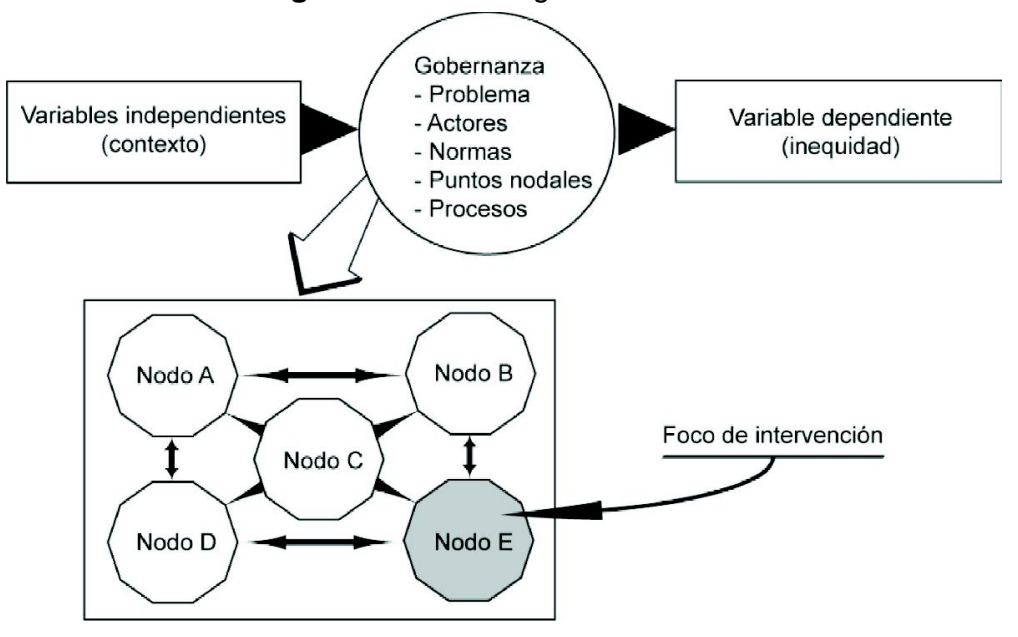

\section{Conclusión}

Este artículo promueve el uso analítico de gobernanza para la investigación en sistemas de salud, también reconoce que existen otras corrientes que incluyen aspectos normativos para su uso en el campo de las políticas de salud. Otros artículos de esta publicación reflejan estas corrientes, promovidas por grupos de actores académicos que adoptan sus propias definiciones de la buena gobernanza y que no se encuadran en el marco de los preceptos promovidos por las agencias internacionales.

A diferencia de los usos normativos, el MAG tiene un gran potencial en materia de investigación para comprender mejor los procesos de formulación e implementación de políticas sociales. Se aplica de manera creciente en el campo de la investigación de políticas y sistemas de salud, pero también en temas ambientales o de seguridad pública. Asimismo, está siendo sometido a pruebas en varios estudios de caso y todavía debe ser considerado como un trabajo en constante evolución.

Espero que este modelo constituya un progreso por su facilidad de uso y su claridad. Por lo menos, intente transformar, a través de criterios rigurosos, el concepto vago y a menudo conflictivo existente en la metodología empírica 
Agradecimientos. A Mariana Cruz por su ayuda con el castellano escrito, a Roberto Bazzani (IDRC), Carmen Rico (UQAM), Ernesto Báscolo y Natalia Yavich (Instituto Lazarte) y al equipo del GREG (Ginebra) para el apoyo y los comentarios.

Conflictos de interés: ninguno

\section{REFERENCIAS}

1. Hufty M, Bascolo E, Bazzani R. Gobernanza en Salud. Un aporte conceptual y analítico para la investigación. Cadernos de Saúde Pública/Reports in Public Health. 2006; 22(x): 109118.

2. Hufty M [Internet]. La gouvernance est-elle un concept opérationnel? Proposition pour un cadre analytique. Fédéralisme-Régionalisme 2007; 7(2). Disponible en: http://popups.ulg.ac.be/ federalisme/document.php?id=635 Consultado: 15 de noviembre de 2009 .

3. Hufty M. L'objet gouvernance. In: Hufty M, Dormeier-Freire P, Plagnat P, Neumann V. Jeux de gouvernance. Regards et réflexions sur un concept. Paris: Karthala-IUED; 2007: 13-28.

4. Peirce CS. How to make our ideas clear. Popular Science Monthly 1978; 12: 286-302.

5. Diccionario de la Real Academia Española. 22a Edición. Madrid: Espasa-Calpe; 2001.

6. Kooiman J. Modern Governance: New Government-Society Interactions. London: Sage; 1993.

7. Camou A. Los desafíos de la Gobernabilidad. México: Flacso-IISUNAM-Plaza y Valdés; 2001.

8. Olsen J. University Governance: Non-participation as Exclusion or Choice. In: March J, Olsen J. (Eds.) Ambiguity and Choice in Organizations. Bergen: Universitetsforlaget; 1976: 277 313.

9. Williamson OE. Transaction-cost Economics: The Governance of Contractual Relations. Journal of Law and Economics 1979; 22(2): 233-261.

10. Williamson OE. The Economics of Governance:Framework and Implications. Journal of Theoretical Economics 1984; 140: 195-223.

11. Williamson OE The Mechanisms of Governance. Oxford: Oxford University Press; 1996: 3-20.

12. OECD. OECD Principles of Corporate Governance. Paris; 2004.

13. Rosenau JN. Governance without Government: Systems of Rule in World Politics. Los Angeles: University of South California; 1987.

14. Krasner SD. Structural Causes and Regime Consequences: Regimes as Intervening Variables. International Organization 1982; 36(2): 185-205.

15. Commission on Global Governance. Our Global Neighbourhood. Oxford: Oxford University Press; 1995. Nuestra traducción.

16. Pierre J, Peters BG. Governance, Politics and the State. New York: St. Martin's Press; 2000

17. Jessop B. The Rise of Governance and the Risks of Failure: the Case of Economic Development. International Social Science Journal, 1998; 155: 29-45.

18. Kooiman J. (ed.). Modern Governance: New Government-Society Interactions. London: Sage; 1993.

19. Rhodes RAW. Understanding Governance: Policy Networks, Governance, Reflexivity and Accountability. Philadelphia: Open University Press; 1996.

20. Holliday I. Is the British State Hollowing Out? Political Quarterly 2000; 71(2): 167-176.

21. Marinetto M. Governing the Centre: A Critique of the Anglo-Governance School. Political Studies 2003; $51:$ 592-608.

22. Hufty M. Aux racines de la pensée comptable. In: Hufty M. (dir.) La pensée comptable: Etat, néolibéralisme, nouvelle gestion publique. Nouveaux Cahiers de l'IUED no 8. GenèveParis: Institut universitaire d'études du développement-Presses Universitaires de France; 1999: 15-40. 
23. World Bank. Governance and Development. Washington: IBRD; 1992. Nuestra traducción. 24. Kaufmann D, Kraay A, Mastruzzi M. Governance Matters IV: Governance Indicators for 19962004. Washington: The World Bank; 2005.

25. Hufty M. L'interdisciplinarité en pratique: un projet de recherche à Madagascar. Desenvolvimento e Meio Ambiente. 2005; 11-12: 23-40.

26. Bourdieu P. Questions de sociologie. Paris: Les Éditions de Minuit; 1984.

27. Searle J. The Construction of Social Reality. New York: The Free Press; 1995.

28. Katzenstein PJ (Ed.). The Culture of National Security: Norms and Identity in World Politics. New York: Columbia University Press; 1996.

29. Haas P. Introduction: Epistemic Communities and International Policy Coordination. International Organization 1992; 46(1): 1-35.

30. Accardo A. Initiation à la sociologie de l'illusionnisme social. Bordeau: Le Mascaret; 1983.

31. Prats J. [Internet]. Gobernabilidad democrática para el desarrollo humano. Marco conceptual y analítico. Disponible en: http://www.hegoa.ehu.es/dossierra/gobernanza/3Prats2001.pdf Consultado 15 de Noviembre 2009. 